\title{
MORE ON THE SU(2) DECONFINEMENT TRANSITION IN THE MIXED ACTION
}

\author{
Rajiv V. Gavai* \\ Theoretical Physics Group, Tata Institute of Fundamental Research \\ Homi Bhabha Road, Mumbai 400005, India \\ Manu Mathur ${ }^{\dagger}$ \\ Dipartimento di Fisica dell' Universitâ and I.N.F.N \\ Piazza Torricelli 2 Pisa-56100, Italy
}

\begin{abstract}
We examine certain issues related to the universality of the $\mathrm{SU}(2)$ lattice gauge theory at non-zero temperatures. Using Monte Carlo simulations and strong coupling expansions, we study the behavior of the deconfinement transition in an extended coupling plane $\left(\beta, \beta_{A}\right)$ around the tricritical point where the deconfinement transition changes from second to first order. Our numerical results on $N_{\tau}=2,4,6,8$ lattices show that the tricritical point first moves down towards the Wilson axis and and then moves slowly upwards, if at all, as the lattice spacing is reduced. Lattices with very large $N_{\tau}$ seem to be therefore necessary for the mixed action to exhibit the critical exponents of
\end{abstract}

\footnotetext{
*Email:gavai@mayur.tifr.res.in

${ }^{\dagger}$ Permanent address: S. N. Bose National Centre for Basic Sciences, JD Block, Sector III, Slat Lake, Calcutta 700064, India. Email:manu@hpth.difi.unipi.it
} 
the three dimensional Ising model for positive values of the adjoint coupling. PACS numbers:11.15Ha

Typeset using REVTEX 


\section{INTRODUCTION}

Confinement of the non-abelian color degrees of freedom has been a challenging problem ever since gauge theories were formulated for quark-gluon interactions. In the beginning, our understanding of such gauge theories followed mainly from perturbation theory. Due to the property of asymptotic freedom, the perturbative approximations are valid for short distance phenomenon but they are inadequate to explore long distance physics like confinement of quarks. A strong evidence for, and a much better insight of, the color confinement mechanism in non-abelian gauge theories has been provided by analytical computations and Monte Carlo simulations of quantum field theories with a non-perturbative lattice regularisation. The simplest of such theory is described by the $S U(2)$ Wilson action [1]. It was expected that non-abelian gauge theories in general do not have any phase transitions separating strong and weak coupling regimes. Therefore confinement, explicitly shown on the lattice in the strong coupling region, should persist also in the continuum limit. However, it was later found [2] that certain non-abelian lattice gauge theories (e.g. $S O(3), S U(4), S U(5)$ ), with Wilson form of action undergo bulk transitions separating strong confining region from the weak coupling region where the continuum limit of the theory exists. Bhanot and Creutz [3], extending the form of the action proposed by Wilson, showed that this apparent loss of confinement can be attributed to lattice artifacts associated with the choice of action, namely the so-called bulk phase transitions.

Subsequent to the work of Bhanot and Creutz to characterize the bulk transitions in the extended coupling plane, important reasons for further exploration of this action in the past have been to study the basic mechanism of confinement itself [4] and to find out the origin of these bulk transitions [5-7]. It has been a common folklore that the abrupt change from the strong coupling region to the scaling region for the Wilson action is due to the proximity of the critical point at the end of the first order line CD in Fig. 1, where the phase diagram obtained in Ref. [3] is shown, and that a "smoother" continuum limit may be obtained by going to negative $\beta_{A}$, the additional coupling for this action. Due to the theoretical 
expectations of the role of $S O(3)\left(S U(N) / Z_{N}\right)$ monopoles in $S U(2)(S U(N))$ confinement [8], the above model is tailor made to study the interplay of these topological degrees of freedom and their role in confinement between $S U(2)(S U(N))$ and $S O(3)\left(S U(N) / Z_{N}\right)$ lattice gauge theories. In fact, the plaquette susceptibility peak in the cross-over region in $S U(2)$ lattice gauge theory and the bulk transition line $B C D$ have been attributed to the underlying $S O(3)$ theory and its first order transition [9, 10]. These issues can be also analyzed and tested by exploring the mixed action at non-zero temperatures and will be further discussed after presentation of our results.

The rich phase diagram associated with the mixed action, shown in Fig. 1 by solid and dashed lines, was established mainly by Monte Carlo simulations on relatively small lattices [3] $\left(4^{4}-5^{4}\right)$ with periodic boundary conditions. Since these small lattices were also at finite temperature, the phase diagram is incomplete in the absence of the deconfinement transition line. Along the $\beta_{A}=0.0$ axis, several finite temperature investigations have shown the presence of a second order deconfinement phase transition. Its critical temperature has been shown [11] to exhibit asymptotic scaling and its critical exponents have been shown 112 to be in very good agreement with those of the three dimensional Ising model. Effective field theory arguments for the order parameter were used by Svetitsky and Yaffe [13] to conjecture the finite temperature $\mathrm{SU}(2)$ gauge theory and the three dimensional Ising model to be in the same universality class. The verification of this universality conjecture thus strengthened our analytical understanding of the deconfinement phase transition. Our work 14,15] on the extended action at non-zero temperatures began with the motivation to locate the line of deconfinement transition in the couplings plane $\left(\beta, \beta_{A}\right)$. Our simulations yielded the following surprising results:

a] The transition remained second order in agreement with the universality conjectured exponents up to $\beta_{A} \approx 1.0$ but it became definitely first order for large enough $\beta_{A}(\geq$ $1.4)$.

b] There was no evidence of a second separate transition at larger $\beta_{A}$, as would be sug- 
gested by the claim of Ref. [3] of a bulk transition there. For $N_{\tau}=4$ lattices the line of deconfinement transition was coincident with the line of bulk transitions of Ref. [3] but for $N_{\tau}=2$ there were no symptoms of any transition at those locations. The line of deconfinement phase transition, on the other hand, did move to smaller $\beta$ for all $\beta_{A}$, as $N_{\tau}$ changed from 4 to 2 .

While the details of our analysis and results can be found in the works cited above, the key findings which lead us to these conclusions were following:

a] The deconfinement order parameter, $\langle L\rangle$ (see next section for definition), acquired nonzero large value at the only transition found on all lattices studied (i.e $N_{\tau}=2,4,6,8$ ) and showed clear co-existence of both phases at the transition point for larger $\beta_{A}$.

b] The same critical exponent which established the transition to be in the Ising model universality class for $0.0 \leq \beta_{A} \leq 1.0$ became equal to the space dimensionality, as a first order deconfinement phase transition would have, for larger $\beta_{A}$.

c] The plaquette susceptibility showed a decrease at $\beta_{A}=1.1$ when the lattice 4 -volume was increased by a factor of 16; it should diverge, i.e., increase 16-fold, if there were a first order bulk phase transition at $\beta_{A}=1.1$.

The plaquette susceptibility results above are very similar to those of Ref. [17 who too found a decrease in it while increasing the lattice volume by a factor of 16 . On increasing the lattice size further, no further finite size dependence was found, leading to a conclusion that the finite size effects on smaller lattices are due to finite temperature effects. While larger lattices will be needed in our case too to see if a similar conclusion is reached, it has to be emphasized that conclusions based on finite size scaling usually do assume that the lattices are large enough for the scaling to set in. Thus a distinguishing feature between the bulk and deconfinement phase transitions, $i$. e. the finite size scaling behavior of the coupling at which the transition takes place with the temporal size of the lattice which leads one to expect the bulk transitions to move much less compared to the deconfinement 
transitions, is not necessarily useful here since it is not clear how big lattices are needed for this behavior to set in at various values of $\beta_{A}$. We have therefore relied heavily on the order parameter $\langle L\rangle$ to label a transition as a deconfinement phase transition, as mentioned in a] above.

Recently, the above surprising results showing the change in the order of the deconfinement transition and the absence of the bulk transition were confirmed 16 for another variant of the $\mathrm{SU}(2)$ action with a Villain form for the adjoint $\mathrm{SO}(3)$ part. We will later comment more on the above action. Taken together, these results pose many questions about the continuum limit of the deconfinement phase transition and about the existence of separate bulk phase transitions. The foremost amongst them is about an apparent qualitative violation of the universality", since an apparently irrelevant coupling seems to change the order of the deconfinement phase transition. The early simulations of the $S U(2)$ lattice gauge theory are known to have yielded quantitative violations of universality [18]. However, attributing them to the ignored higher orders in $g_{u}^{2}$, it has been shown [19 21] that dimensionless ratios of physical quantities have much weaker such violations. Indeed, one can hope that these violations will smoothly disappear under the error bars of the simulations as the cut-off becomes smaller. This will obviously not be the case for any qualitative violations of universality. Of course, the region of couplings where universal results are obtained may have such an irregular shape that still larger lattices are needed to obtain universal results. It is not clear in that case, however, what the universal result would be. Clearly, if a universal result exists in the $a \rightarrow 0$ limit, then the tricritical point $\mathrm{T}$, where the deconfinement phase transition changes order, must not appear on any renormalisation group flow lines to the $g_{u}=0.0$ critical point and must thus be invisible in the continuum limit. If the point $\mathrm{T}$ moves up to large positive $\beta_{A}$ with increasing $N_{\tau}$ (and decreasing lattice spacing $a$ ), then the

\footnotetext{
${ }^{1}$ Note that this universality, which results from the freedom of choice of the lattice type and action, is different from the finite temperature universality of critical exponents discussed earlier.
} 
$\mathrm{SU}(2)$ deconfinement phase transition could still be of second order in continuum limit with Ising model exponents. This would be so irrespective of $\beta_{A}$ used for simulations. If, on the other hand, the point $\mathrm{T}$ moves to large negative $\beta_{A}$, the universality with Ising model will be lost and the transition will be first order, again irrespective of $\beta_{A}$ used in simulations.

It may be argued that the presence of a line of bulk phase transitions and its end point will strongly modify the approach to continuum limit and thus large lattices are mandatory for seeing the universal physics at large $\beta_{A}$. It needs to be noted therefore that the bulk line in question was established only in numerical simulations on small, $4^{4}-5^{4}$ lattices. A recent simulation 22 at $\beta_{A}=1.25$ on larger $N^{4}$ lattices, with $N=6,8,10,12$ and 16, found 1) a linear decrease in the average discontinuity in plaquette, $\Delta P$, with $N$ and 2) a plaquette susceptibility exponent of $2.09 \pm 0.31$ in contrast to the expected value of 4 for a first order bulk phase transition. This suggests that the end point of the bulk line is at $\beta_{A}>1.25$. This is explicitly shown in Fig. 1 by drawing solid and dashed bulk lines above and below $\beta_{A}=1.25$ respectively. While the result of Ref. [22] does explain the above mentioned results on plaquette susceptibility, the mystery of the apparent coincidence of the two different transition lines still remains for larger $\beta_{A}$. As we will show below, the deconfinement phase transition for $N_{\tau}=4$ lattices, as identified by the order parameter, $\langle|L|\rangle$, turns first order already at $\beta_{A}=1.25$, suggesting that the bulk line or its end point are unlikely sources of this change.

In this paper, we address the issue of the trajectory of point $T$ with decreasing lattice spacing $a$, after defining in the next section the action we investigate and the observables we use along with their scaling laws. A simple strong coupling calculation is presented in Section 3, which suggests that the point $\mathrm{T}$ moves up in the plane to infinity. However, in our numerical simulations, described in Section 4, we find that it moves down on going from $N_{\tau}=2$ to 4 . On increasing $N_{\tau}$ further to 6 and then 8 , we observe a very small upward movement by comparing the relative shapes of the Polyakov loop histograms. The last section contains a brief summary of our results and their discussion. 


\section{THE MODEL AND THE OBSERVABLES}

The lattice action is constrained only by a) the gauge invariance and b) the limit of zero lattice spacing which must coincide with the continuum form of the action. Infinitely many different forms satisfying these criteria can be written down. Bhanot and Creutz extended the Wilson action to a form described by the action,

$$
S=\sum_{P}\left(\beta\left(1-\frac{1}{2} \operatorname{Tr}_{F} U_{P}\right)+\beta_{A}\left(1-\frac{1}{3} \operatorname{Tr}_{A} U_{P}\right)\right) \quad .
$$

Here $U_{P}$ denotes the directed product of the basic link variables which describe the gauge fields, $U_{\mu}(x)$, around an elementary plaquette $P . F$ and $A$ denote that the respective traces are evaluated in fundamental and adjoint representations respectively. Comparing the naive classical continuum limit of eq. (1) with the standard $S U(2)$ Yang-Mills action, one obtains

$$
\frac{1}{g_{u}^{2}}=\frac{\beta}{4}+\frac{2 \beta_{A}}{3}
$$

Here $g_{u}$ is the bare coupling constant of the continuum theory. Introducing another coupling $\theta$, defined by $\tan \theta=\beta_{A} / \beta$, the asymptotic scaling relation [23] for this action is

$$
a=\frac{1}{\Lambda(\theta)} \exp \left[-\frac{1}{2 \beta_{0} g_{u}^{2}}\right]\left[\beta_{0} g_{u}^{2}\right]^{\frac{-\beta_{1}}{2 \beta_{0}^{2}}},
$$

where

$$
\log \frac{\Lambda(0)}{\Lambda(\theta)}=\frac{5 \pi^{2}}{11} \frac{6 \tan \theta}{(3+8 \tan \theta)} .
$$

Here $\beta_{0}$ and $\beta_{1}$ are the usual first two universal coefficients of the $\beta$ function for the $S U(2)$ gauge theory: they do not depend on $\theta$.

One sees clearly from the equations above that the introduction of a non-zero $\beta_{A}$, leads merely to a different $g_{u}$ and a correspondingly different value for the scale $\Lambda(\theta)$. However, each of these theories, including the usual Wilson theory for $\beta_{A}=0.0$ flow to the same critical fixed point, $g_{u}^{c}=0$, in the continuum limit and has the same scaling behavior near the critical point. The different forms of action, obtained by varying $\beta_{A}$, are simply related 
by a redefinition of coupling constant and the intrinsic scale $\Lambda$ and yield the same universal continuum physics. Numerical investigations for different $\beta_{A}$ thus constitute a necessary check of the finite cut-off effects in the non-perturbative results obtained for $\beta_{A}=0.0$, i.e., the Wilson action.

Bhanot and Creutz [3] found that the lattice theory defined by the extended action has a rich phase structure (Fig. 1). Along the $\beta=0$ axis it describes the $S O(3)$ model which has a first order phase transition at $\beta_{A}^{\text {crit }} \sim 2.5$. At $\beta_{A}=\infty$ it describes the $Z_{2}$ lattice gauge theory again with a first order phase transition at $\beta^{\text {crit }}=\frac{1}{2} \ln (1+\sqrt{2}) \approx 0.44$ [24]. Ref. [3] found that these first order transitions extend into the $\left(\beta, \beta_{A}\right)$ plane, ending at an apparent critical point located at $(1.5,0.9)$. These transition lines are shown in Fig. 1 by continuous lines. Using finite size scaling, Ref. [22] has recently shown that the critical endpoint must have $\beta_{A} \geq 1.25$. More simulations on larger lattices will be required to determine the endpoint precisely. The qualitative aspects of this phase diagram were also reproduced by mean field theory [5] and large $\mathrm{N}$ [6] and strong coupling [7] expansions.

Simulations of the mixed action above at finite temperature are made on asymmetric $N_{\sigma}^{3} \times$ $N_{\tau}$ lattices, with periodic boundary conditions in the (shorter) $\tau$-direction. The partition function at finite temperature is given by,

$$
Z=\int \prod_{x, \mu} d U_{\mu}(x) \exp (-S)
$$

The order parameter for the deconfinement transition is the Polyakov loop [25] defined by

$$
L(\vec{n})=\frac{1}{2} \operatorname{Tr} \prod_{\tau=1}^{N_{\tau}} U_{0}(\vec{n}, \tau),
$$

Here $U_{0}(\vec{n}, \tau)$ is the time-like link at the lattice site $(\vec{n}, \tau)$. Due to periodic boundary condition in the time-like direction at finite temperature the action of eq. (1) has a $Z_{2}$ invariance corresponding to the center of the gauge group. Defining this symmetry to be

$$
U_{0}\left(\vec{n}, \tau_{0}\right) \rightarrow z U_{0}\left(\vec{n}, \tau_{0}\right) \quad \forall n, \quad \tau_{0}: \text { fixed }, \text { and } z \in Z_{2}
$$

one sees that under its transformation the Polyakov loop changes by 


$$
L \rightarrow z L \quad
$$

while the action in eq. (11) remains unchanged.

A non-vanishing value for $\langle L\rangle$, with respect to the partition function in eq. (5), signals a spontaneous break-down of the global $Z_{2}$ symmetry. $\langle L\rangle$ is also an order parameter for the deconfinement phase transition, as it (or equivalently its average value $L=\frac{1}{N_{\sigma}^{3}} \sum_{\vec{n}} L(\vec{n})$ ) can also be shown to be a measure of the free energy of an isolated free quark [25]. In order to monitor the critical behavior of the deconfinement transition, we also define the Polyakov loop susceptibility:

$$
\chi_{N_{\sigma}}=N_{\sigma}^{3}\left(\left\langle L^{2}\right\rangle-\langle L\rangle^{2}\right)
$$

In the thermodynamic limit, a second order transition is characterized by the following critical exponents:

$$
\begin{array}{ccc}
\langle L\rangle \propto\left|T-T_{c}\right|^{\beta} & \text { for } & T \rightarrow T_{c}^{+} \\
\chi \propto\left|T-T_{c}\right|^{-\gamma} & \text { for } & T \rightarrow T_{c} \\
\xi \propto\left|T-T_{c}\right|^{-\nu} & \text { for } & T \rightarrow T_{c} .
\end{array}
$$

Here $\xi$ is the correlation length corresponding to the Polyakov loop correlations and $\beta \approx 0.325, \gamma \approx 1.24$ and $\nu \approx 0.63$ are the Ising model exponents, assuming the universality conjecture to be true. The best determination of these exponents for the $S U(2)$ lattice gauge theory was made [12] by using the finite size scaling theory [26], according to which, the peak of the $L$-susceptibility on a lattice of spatial extent $N_{\sigma}$ is expected to grow like

$$
\chi_{N_{\sigma}}^{\max } \propto N_{\sigma}^{\omega}
$$

where $\omega=\gamma / \nu=1.97$. If the phase transition were to be of first order instead, then one expects the exponent $\omega=3$, corresponding to the dimensionality of the space [27]. In addition, of course, the order parameter is expected to exhibit a sharp, or even discontinuous, jump and the corresponding probability distribution should show a double (multi) peak 
structure. For $\beta_{A}=0$, the universality prediction was verified by Monte Carlo simulation by Engels et. al. [12], who found $\omega=1.93 \pm 0.03$, whereas we found [15] $\omega=3.25 \pm 0.24$ for $\beta_{A}=1.4$ on $N_{\tau}=2$ lattices.

\section{STRONG COUPLING}

Before turning to the results of our simulations to determine $\omega$ and to locate the tricritical point, it may be an instructive exercise to find out what hints the strong coupling expansion can provide. Such expansions for the free energy [28,29] and string tension [30] have been used in the past to study $S U(N)$ deconfinement transition for the Wilson action. The basic strategy is to obtain an effective potential for the order parameter $L$, by expanding the partition function in powers of the inverse coupling constant(s) and integrating out the spatial links . Due to the $Z_{2}$ symmetry of the theory, the Landau-Ginzburg effective action is an even polynomial in the Polyakov loop for the $S U(2)$ theory. To lowest order:

$$
\begin{aligned}
S_{e f f} & =-\frac{1}{2} \sum_{\vec{n}} \log \left(1-L^{2}(\vec{n})\right)-4\left(\frac{\beta}{4}\right)^{N_{\tau}} \sum_{\vec{n}, i} L(\vec{n}) L(\vec{n}+i) \\
& -\left(\frac{\beta_{A}}{9}\right)^{N_{\tau}} \sum_{\vec{n}, i}\left(4 L^{2}(\vec{n})-1\right)\left(4 L^{2}(\vec{n}+i)-1\right)
\end{aligned}
$$

Here the first term is independent of the couplings and is the exact Jacobian due to the change of the temporal link variables to $L(\vec{n})$ after all the link integrations. The last two terms are the leading strong coupling terms in $\left(\frac{\beta}{2}\right)$ and $\left(\frac{\beta_{A}}{3}\right)$ with the assumption that both $\frac{\beta}{2}$ and $\frac{\beta_{A}}{3}$ are small and treated on the same footing. Only the leading order terms in $\beta$ and $\beta_{A}$ are retained in the effective action here. Demanding translational invariance for the configuration which minimizes the action, one can easily obtain the effective potential for the order parameter. Expanding the log term for small $L$, one has the following results for the coefficients, $b_{2}, b_{4}$ and $b_{6}$ of the $L^{2}, L^{4}$ and $L^{6}$ terms in the effective potential:

$$
V_{e f f}(L)=b_{2} L^{2}+b_{4} L^{4}+b_{6} L^{6}
$$


where

$$
\begin{aligned}
& b_{2}=\frac{1}{2}-12\left(\frac{\beta}{4}\right)^{N_{\tau}}+24\left(\frac{\beta_{A}}{9}\right)^{N_{\tau}} \\
& b_{4}=\frac{1}{4}-48\left(\frac{\beta_{A}}{9}\right)^{N_{\tau}} \\
& b_{6}=\frac{1}{6} .
\end{aligned}
$$

The positivity of the $b_{6}$-term (and all other higher terms) ensures that the effective potential is bounded from below. Note that for small enough $\beta$ and $\beta_{A}, b_{2}$ and $b_{4}$ are also positive, favoring thus the confined phase of $L=0$. For small $\beta_{A}$ and arbitrary $\beta, b_{4}$ remains positive but $b_{2}$ goes through a zero, giving rise to a second order phase transition at a critical $\beta$ obtained by setting $b_{2}$ to zero. As $\beta_{A}$ increases, $b_{4}$ becomes negative above a critical value of $\beta_{A}$. The effective potential then has two additional minima in addition to the one at $L=0$. As $\beta$ increases, these minima deepen and become equal to the one at $L=0$, yielding a first order deconfinement phase transition. The tricritical point, where the deconfinement phase transition changes to become first order, is given by setting the coefficients $b_{2}$ and $b_{4}$ to zero.

In this leading order strong coupling expansion, the tricritical points are $\left(\beta^{\text {tricrit }}, \beta_{A}^{\text {tricrit }}\right)$ $=(0.913,0.649),(1.91,2.418)$ for $N_{\tau}=2$ and 4 lattices respectively. This suggests that the tricritical point moves towards the top right corner $\left(\beta=\infty, \beta_{A}=\infty\right)$ of the phase diagram as the lattice spacing is reduced. Thus the the tricritical point will not seen by the continuum limit. Of course, one needs to improve the leading order strong coupling result as $N_{\tau}$ increases and check that this conclusion remains unchanged. Nevertheless, these results are encouraging for two reasons. Firstly, they provide a concrete example of how the $S U(2)$ gauge theory at finite temperature can have a first order phase transition. Indeed, it should be noted that the bulk phase transition plays no role above in changing the order of the deconfinement phase transition at large $\beta_{A}$. Secondly, the qualitative trend suggested by this simple exercise is in agreement with the naive idea of independence of physical results with respect to irrelevant couplings. Of course, the key question of the limit of the coefficients $b_{i}$, 
as the lattice spacing $a \rightarrow 0$, can only be resolved by simulations at present and, in principle, the trajectory of the tricritical point could go either way in that limit. In the next section, we describe the results of our simulations which were made in an attempt to answer this issue.

\section{RESULTS OF THE SIMULATIONS}

Our Monte Carlo simulations were done using Metropolis algorithm on $N_{\sigma}^{3} \times N_{\tau}$ lattices with $N_{\tau}=2,4,6,8$ and $N_{\sigma}=8,10,12,16$. The many different values of $N_{\sigma}$ were chosen to study the finite size scaling behavior of the theory and to compute the critical exponent $\omega$, while the $N_{\tau}$ values were chosen to monitor the movement of the tricritical point with decreasing lattice spacing. We also used $N_{\sigma}=16$ in one case to be sure of the critical exponent. The possible ranges for the tricritical points for different $N_{\tau}$ were known from our earlier work, and the simulations were carried out at $\beta$ and $\beta_{A}$ in these ranges. Histogramming techniques were used to extrapolate to nearby $\beta$ values while estimating the height and location of the peak of various susceptibilities. The values of the critical exponents from our earlier simulations 14,15 on $N_{\tau}=2,4$ lattice are summarized in Table 1, where the result of Ref. 12] for $\beta_{A}=0.0$ is also given. One sees a good agreement with the Ising model exponent for $N_{\tau}=4$ for $\beta_{A} \leq 0.9$, suggesting that the tricritical point must lie at higher $\beta_{A}$ in this case. On the other hand, no check of the universality with the three dimensional Ising model has so far been made for the $N_{\tau}=2$ lattices: the corresponding $\omega$ is unknown although all earlier simulations do indicate a continuous transition. From our previous work [15], we know that the transition for $N_{\tau}=2$ and for $\beta_{A} \geq 1.4$ is a strong first order one. For $\beta_{A}=1.4$, the exponent $\omega=3.25(24)$, with a typical tunneling time of $\approx 30,000-40,000$ Monte Carlo

sweeps. Thus the effective ranges for the $\beta_{A}$ of the tricritical points were $1.4>\beta_{A}^{\text {tricrit }}$ for $N_{\tau}=2$, and $\beta_{A}^{\text {tricrit }}>0.9$ for $N_{\tau}=4$. No firm upper bound was known for the latter case, although we had good indications that $1.5>\beta_{A}^{\text {tricrit }}$, as Ref. [14] found a co-existing two state signal in both the Polyakov loop, $L$, and the plaquette, $P$, at $\beta_{A}=1.5$. 
In our earlier work, the simulations at $\beta_{A}=1.1$ on the $N_{\tau}=2$ lattices did not reveal a clear three peak structure in the histogram of the Polyakov loop on the $N_{\sigma}=8,10,12$ lattices, although the peaks did become a bit sharper on going to the $N_{\sigma}=12$ lattice. Correspondingly the determination of the critical exponent did not fix the order of the transition uniquely. This is similar to the $N_{\tau}=4$ results at $\beta_{A}=1.1$ [14] where the histograms and the evolution graphs of the Polyakov loop gave a very weak two state signal with an $\omega \simeq 2.34$, lying between values characteristic of first and second order phase transition. Such a behavior of the deconfinement transition can be understood from the point of view of the effective potential in terms of the Polyakov loop, if these simulations were indeed close to the tricritical point. As argued in earlier section, the first two leading coefficients of $L^{2}$ and $L^{4}$ terms of the effective potential are then close to being zero, leading to a reasonably flat effective potential around $L=0.0$, a fact which we will later exploit to conclude about the movement of the tricritical point as the lattice spacing is reduced. However, as a consequence, much larger statistics is required to sample the exact nature of the effective potential near the tricritical point to separate a weak first order transition from a second order one. We therefore increased the statistics to typically $4 \times 10^{6}$ sweeps to compute the critical exponents and focused more on $\beta_{A}$ close to 1.1. We, however, also made simulations on $N_{\tau}=2$ lattices at $\beta_{A}=0.0,0.8$, and 0.9 to determine the critical exponent $\omega$ and thus the range for the tricritical point more precisely. The observables were typically recorded after every 20 sweeps to reduce auto-correlation. The errors were estimated by further binning the data and the typical bin size was $O(100)$.

Figs. $2 \mathrm{a}, 2 \mathrm{~b}, 2 \mathrm{c}$ and $2 \mathrm{~d}$ exhibit the results for $L$-susceptibility for the $N_{\tau}=2$ lattices for $\beta_{A}=0.0,0.8,1.1$ and 1.25 respectively. The results for $\beta_{A}=0.9$ are similar to those in Fig. 2c and are therefore not shown here. In each of these figures, the range of expected values for the peak height for the $N_{\sigma}=10$ and 12 lattices is also shown by two horizontal lines by assuming the validity of eq. (13),$\omega=1.97$, and by using $\chi_{N_{\sigma}=8}^{\max }$ for each case. The errors on the respective $\chi_{N_{\sigma}=8}^{\max }$ induce the spread between the lines. We always chose a fresh starting point in an iterative manner, if the initial guess was too far away from the extrapolated 
estimate for the location of the peak. This reduced the influence of the unknown systematic errors in the peak height due to our extrapolation procedure. As seen in Figs. 2a-2d, we do hope that this source of errors has been brought under control by our choice of the simulation points, and that it does not annul our conclusions about whether the critical exponent is close to 3 or 1.97 .

The independence of $\omega$ for $\beta_{A}=0.0$ (and small $\beta_{A}$ ) for $N_{\tau}=2$ and 4 and its agreement with 3-d Ising model value is satisfying since there is only one known critical point in $Z(2)$ symmetric theories and a priori one expects the exponents to be universal. Moreover, strong coupling arguments, which predict a second order phase transition for small $\beta_{A}$, should be more reliable for smaller $N_{\tau}$. Quantitatively, however, one notices the leading order strong coupling prediction for $\beta^{\text {crit }}\left(N_{\tau}=2\right)=0.816$ for $\beta_{A}=0.0$ to be far away from the corresponding Monte Carlo determination. Furthermore, its $\beta_{A}$-dependence seems to be also in the wrong direction. Thus, one really could have expected surprises in form of a qualitative difference from the strong coupling prediction as well. On the other hand, it may be more natural to expect the effect of higher orders in quantitative shifts and not in qualitative features. Since it is unclear whether $N_{\tau}=4$ is in the strong coupling region, the universality conjecture for the critical exponents needs to be tested on lattices with larger $N_{\tau}$ and thus closer to the continuum limit even for the Wilson action, i.e, $\beta_{A}=0.0$.

The values of the critical coupling, $\beta^{\text {crit. }}$ and the finite size scaling exponent, $\omega$, obtained by fitting the peak heights to eq.(13), are given in the Table 2 for all the $\beta_{A}$ values we investigated, including $\beta_{A}=0.9$. These estimates of $\omega$ in Table 2 , along with the agreement in Fig. 2 with the predictions based on a $\omega=1.97$ show the deconfinement phase transition for $N_{\tau}=2$ lattices to be a clear second order with Ising model exponents for $1.25 \geq \beta_{A} \geq 0.0$ . At $\beta_{A}=1.25$, additional simulations were performed on $N_{\sigma}=16$ to confirm the second order nature of the transition. Note that if the predictions for $\omega=3$, corresponding to a first order phase transition were to be displayed in Figs. 2 then they would overshoot by a large amount, especially in Fig. $2 d$ where they would be too big by a factor of 2 for the $N_{\sigma}=16$ lattice. Also interesting to note is the resolution of the ambiguity in establishing the order of 
the phase transition for $\beta_{A}=1.1$ in these better statistics simulations. The finite size scaling exponent is $\omega=1.79(02)$ and is thus a lot closer to the Ising value. The tricritical point $\mathrm{T}$ on a $N_{\tau}=2$ lattice is thus constrained to lie in the range $1.4 \geq \beta_{A}>1.25$, as indicated in Fig. 1 by the gap between the filled circles for the first order transition points and hollow circles for the second order transition points. Thicker dashed and dotted lines show the first order and second order deconfinement phase transition lines.

Since the exponent $\omega$ was found [14] to be 2.31(28) for $N_{\tau}=4$ at $\beta_{A}=1.1$ and the histograms of the Polyakov loop signaled a very weak first order transition, we chose to re-investigate the transition first at $\beta_{A}=1.1$ and then move to larger values of $\beta_{A}$. Fig. 3 shows the evolution of both $L$ and $P$ at $\beta_{A}=1.25$ on $N_{\sigma}=8,10,12$ lattices. These figures clearly show the coexistence of two states at the deconfinement transition, since $\langle L\rangle \simeq 0$ for one of the phases while it is nonzero and large for the other. The plaquette, $\mathrm{P}$, has a discontinuity at the same location, and further the number of tunnelings and duration in each phase do indeed decrease as the spatial volume increases. Figs. 4a and 4b show the Polyakov loop susceptibility at $\beta_{A}=1.1$ and 1.25 on $N_{\tau}=4$ lattice for various spatial volumes. As in Figs. 2, the expected peak heights for the bigger two lattices are shown by horizontal bars. The only difference here is that the solid horizontal lines in Fig. 4-b show the predicted $\chi_{N_{\sigma}=10,12}^{\max }$ by assuming $\omega=3.0$, while the broken lines in Fig. $4 \mathrm{~b}$ and the solid lines in Fig. 4a are for the Ising value 1.97. The values of couplings at which simulations were performed along with the critical values and the fitted $\omega$ are given in Table 3 . The most astonishing result is that the transition at $\beta_{A}=1.25$ is a first order transition with $\omega=3.13(01)$. This needs to be contrasted with i) the results for $N_{\tau}=2$ and in particular, the Fig. 2d and ii) the results of Ref. [22] where a clear absence of a first order bulk phase transition at $\beta_{A}=1.25$ was shown.

These simulations thus indicate that the tricritical point for $N_{\tau}=4$ lies definitely below $\beta_{A}=1.25$ whereas the corresponding $N_{\tau}=2$ point is definitely above $\beta_{A}=1.25$. This is also clearly seen in Fig. 1, where again the first order transition points for $N_{\tau}=4$ are shown by filled squares, the second order points by hollow squares and the gap between them is 
the allowed range for the tricritical point. As one can see, the tricritical point does shift as the temporal lattice size increases from 2 to 4 . However, the direction of the shift is almost orthogonal to the strong coupling prediction of the previous section and its magnitude is also much smaller. The shift, on the other hand, suggests a possible lack of any correlation of the bulk transitions, if any, with the key observation of the change of the order of the deconfinement phase transition. This is so since any possible bulk transition for $\beta_{A} \leq 1.25$ is definitely not [22] a first order phase transition, making it an unlikely cause of the behavior seen in Figs. 3 and 4. A plausible explanation then is indeed the phenomenon seen in the strong coupling calculations in Sec. 3, i.e., a change of sign in the coefficient $b_{4}$ of the effective potential for the order parameter, $L$.

The above downward movement of $\mathrm{T}$ in the extended coupling plane is puzzling. It calls for a more detailed cross-check on the 3-d Ising model universality, especially for positive values of $\beta_{A}$ but also perhaps for larger $N_{\tau}$ at $\beta_{A}=0.0$. Note that the latter has so far been demonstrated to a very good accuracy only on the $N_{\tau}=2,4$ lattices. Therefore, we decided to monitor the deconfinement transition further by simulating the model on $N_{\tau}=6$ and $N_{\tau}=8$ lattices with $N_{\sigma}=12$ and 16 respectively. On these lattices the simulations were performed at $\beta_{A}=1.1$ and 1.25 to determine the range in which $\mathrm{T}$ may lie. At $\beta_{A}=1.25$ on $N_{\tau}=6$ lattice we found the transition to be first order. The corresponding histogram is plotted in Fig. 5 at $\beta=1.2184$. The three peaks are clearly visible and distinct, though not of equal height. This figure suggests the transition point to be at a slightly higher value of $\beta$ than 1.2184. In choosing this $\beta$ we were guided by the location of the peak of the $L$-susceptibility to locate the critical point. We have found that this criterion to determine the critical point differed a little from that of the effective potential picture (i.e, the nature of the $L$-histograms), although they will coincide in the thermodynamic limit. Comparing the shapes of the histograms at $\beta_{A}=1.1$ in Figs. 6-a,b,c $\left(N_{\tau}=4,6,8\right.$ respectively $)$ on the other hand, one observes that their profiles at the peak tend towards a Gaussian behavior as one makes the lattice spacing smaller by going from $N_{\tau}=4$ to 6 to 8 . This is the expected behavior if the tricritical point shifts upwards from $\beta_{A} \simeq 1.1$ for $N_{\tau}=4$. Since the effective 
potential (14) will have $b_{2} \approx b_{4} \approx 0$ at the tricritical point, it will have a reasonably flat bottom, causing the very flat top for the $N_{\tau}=4$ histogram. In Fig. 6 -a we have plotted the histograms for $N_{\tau}=4$ and $N_{\sigma}=8,10,12$ to confirm the closeness of the tricritical point with the run point, i.e. $\beta=1.327$ and 1.32685 . Moreover, one clearly sees that the flatness is not a finite $N_{\sigma}$ artifact. The reduction in the above flatness would signal $b_{4}$ becoming nonzero, as in a typical second order transition. More quantitatively, the fluctuations in the Polyakov loop at $\beta_{c}=1.327$ on the $N_{\tau}=4$ lattice, are in the range $\Delta L \approx \pm 0.3$, while $\Delta L \approx \pm 0.1$ on the $N_{\tau}=6$ lattice at $\beta_{c}=1.339$, with a reduction by a factor of 3 in the flatness of the histogram at the top. The statistics for the above runs on $N_{\tau}=4$ and 6 lattices was roughly $2 \times 10^{6}$ and $7 \times 10^{6}$ respectively. In principle, this could be taken as a hint that the tricritical point has moved up. However, one knows that $L$ measures the free energy of a point-like test charge and thus has $N_{\tau}$-dependent corrections. These reduce the value of $L$ just above the phase transition as $N_{\tau}$ increases. A perturbative estimate of these corrections will presumably still not yield the factor of two but unless it is quantitatively so demonstrated one is handicapped in drawing a firm conclusion about lack of the finite $N_{\tau}$ effects in the flatness of the effective potential. The Polyakov loop histogram in Fig. 6-c corresponding to $N_{\tau}=8$ has no indication of the co-existence of 2 phases and therefore no evidence of a tricritical point in its vicinity. In this figure we have plotted 2 histograms corresponding to $\beta=1.35$ and 1.3508 showing the the sharp behavior of the transition. This indicates that the qualitative behavior of the histogram changes drastically with the coupling. This again is not expected near the tricritical region. The above drastic reduction in the flat region compared to $N_{\tau}=4$ lattice could again be partially due to the same cause as above. Nevertheless, these qualitative observations suggest that that the tricritical point T moves upwards as $N_{\tau}$ is increased from 4 to 6 and then from 6 to 8 . Even assuming that this upward motion of the the tricritical point continues, one will need a lot larger lattices to confirm universality for $\beta_{A}>0$ since the upward movement is rather small.

\section{SUMMARY AND DISCUSSION}


We simulated the extended action of eq.(11) on $N_{\tau}=2,4,6$ and 8 lattices with varying spatial sizes and determined the order of the deconfinement phase transition by obtaining the critical exponent $\omega$ using finite size scaling theory and also by observing the behavior of the histograms. For $\beta_{A}=0.0$ and $N_{\tau}=2$, we find $\omega=1.92 \pm 0.01$ which is in good agreement with the corresponding Ising model value $1.97 \pm 0.03$. Thus the deconfinement phase transition for the Wilson action for both $N_{\tau}=2$ and 4 is of second order and is in the same universality class as the three-dimensional Ising model. Our simulations show this universality to persist when $\beta_{A}$ is turned on. For the $N_{\tau}=2$ lattice, this is true for at least $\beta_{A} \leq 1.25$ while for $N_{\tau}=4$ the phase transition at $\beta_{A}=1.25$ is already of first order, with $\omega=3.13 \pm 0.01$. The tricritical point where the deconfinement phase transition changes it order is therefore definitely above $\beta_{A}=1.25$ for $N_{\tau}=2$ while it is definitely below $\beta_{A}=1.25$ for $N_{\tau}=4$ lattices. Placing it in the middle of the ranges we have narrowed down, it shifts vertically downwards by about 0.15 . There is a horizontal positive shift in $\beta$, of the order of 0.09 , as well.

The comparison of the above shift of the tricritical point on $N_{\tau}=2$ and 4 lattices with the leading order strong coupling prediction shows surprises. It is, of course, reassuring that the change of the order and the existence of tricritical points for each $N_{\tau}$ is as predicted. However, the predictions do very poorly on a quantitative level. In particular, the directions of the shift of the phase transition as $\beta_{A}$ is turned on, and more importantly, the predicted vertically upward shift of the critical point are in complete contrast with the simulation results for $N_{\tau}=2$ and 4 . On the other hand, the leading order strong coupling equations are known to fail quantitatively for the Wilson theory for both $N_{\tau}=2$ and 4 as well. Putting $\beta_{A}=0.0$ in eq. (18), we recover 28] the criticality condition for the Wilson action : $\beta^{\text {critical }}=4(1 / 24)^{\frac{1}{N_{\tau}}}$. Therefore, the values of the critical couplings to this order on $N_{\tau}=2$ and 4 lattices are 0.816 and 1.807 respectively. These should be compared with the corresponding Monte Carlo values of this work $\left(\beta_{c}\left(N_{\tau}=2\right)=1.88(01)\right)$ and of Ref. 12 $\left(\beta_{c}\left(N_{\tau}=4\right)=2.30(01)\right)$. It would be interesting to check whether the inclusion of higher orders in the strong coupling expansion can yield a better agreement with the simulation 
results, especially for the direction of the vertical shift of the tricritical point.

The above downward movement of the tricritical point on going from $N_{\tau}=2$ to 4 was also observed in Ref. [16] where the Villain form of eq. (11) was simulated. This unexpected behavior of the tricritical point may therefore need to be taken seriously. If this trend persists even on larger lattices then the continuum limit of $\mathrm{SU}(2)$ lattice gauge theory could correspond to a first order deconfinement transition. Hence we simulated this model on larger lattices with $N_{\tau}=6$ and 8 and found some hints of an upward movement of the tricritical point by comparing the shapes of the Polyakov loop histograms. While it unfortunately is not very conclusive, it is encouraging that the trajectory of the tricritical point could potentially be turning up in the coupling plane for these $N_{\tau}$. The tricritical point for both these lattices was still found to be below $\beta_{A}=1.25$ and above $\beta_{A}=1.1$. Much more computational resources on bigger lattices with $N_{\tau}$ much bigger than 8 are necessary to confirm this.

It may therefore be important to understand and explain the origin of the change in the order of the deconfinement transition even away from the continuum, especially since the results of Ref. [22 suggest a lack of a first order bulk phase transition at $\beta_{A}=1.25$ for this action and thus make it implausible that a bulk transition is responsible for such a change. To answer this question, we plan to consider the Villain form for the $\mathrm{SO}(3)$ part of the extended action 4 :

$$
Z=\sum_{\sigma_{p}(n)= \pm 1} \int \prod_{\mu, n} d U_{\mu}(n) \exp \left[\sum_{p}\left(\frac{\beta}{2}+\frac{\beta_{A}}{3} \sigma_{p}\right) \operatorname{Tr}_{F} U_{p}+\lambda \sigma_{p}\right]
$$

Here $\sigma_{p}$ is a $Z_{2}$ plaquette field and the summation over it ensures the invariance of the second term above under $U_{\mu}(n) \rightarrow-U_{\mu}(n)$. For $\lambda=0$, this action is again in the same universality class as that of (1). In fact as already mentioned, its simulations [16] on $N_{\tau}=2,4$ lattices led to exactly the same behavior of the tricritical point as reported in this paper. Besides computational advantages [16], the theoretical advantage of this action is that unlike eq.(四), the $S O(3)$ monopoles and their dynamics is manifest in the form of the $Z_{2}$ plaquette field. The $\mathrm{SO}(3)$ monopole charge density is given by $\prod_{p \in c} \sigma_{p}$; here the product is over the 6 faces of 
a cube [9]. The last term in the above equation is the potential energy for these topological degrees of freedom. In the extreme $(\lambda \rightarrow \infty)$ case when all the $S O(3)$ monopoles are suppressed $\left(\forall \sigma_{p}=+1\right)$, the above extended action reduces to Wilson action with redefined coupling and therefore has only a second order deconfining transition on small $N_{\tau}$ lattices. Therefore, these topological degrees of freedom may have a crucial role in changing the order of the transition. In the extended coupling plane these monopoles cost less and less energy as the adjoint coupling is increased with decreasing values of $\beta$. Therefore, above $\beta_{A}^{\text {tricritical }}$ they might condense giving rise to a first order transition. This can be checked by simulating the above model. If true, this phenomenon will be particularly appealing because precisely the same degrees of freedom and their condensation have been attributed to the first order nature of the 'bulk transition' which we find to be first order deconfinement transition. It may thus also enable in resolving the physical nature of the transition.

\section{ACKNOWLEDGMENTS}

The computations reported here were performed on the DEC Alpha machines of the Tata Institute of Fundamental Research, Bombay and the Theoretical Physics Institute, University of Minnesota, Minneapolis. We would like to thank the staff at these institutes for their support. One of us (R.V.G.) gratefully acknowledges the hospitality he received in TPI, Minneapolis, especially from Profs. J. Kapusta and L. McLerran. This work was supported by the U. S. Department of Energy under the grant DE-FG02-87ER40328. M.M acknowledges the I.N.F.N. fellowship. We wish to thank Prof. M. Grady, SUNY, Fredonia, for pointing out an error in the earlier version of the manuscript. 


\section{REFERENCES}

[1] K. Wilson, Phys. Rev. D10 (1974) 2445.

[2] G. Halliday, A. Schwimmer, Phys. Lett. 101B (1981) 327 and B. Lautrup, Phys. Rev. lett 47 (1981) 9, M. Creutz, Phys. Rev. Lett. 46 (1981) 1441.

[3] G. Bhanot and M. Creutz, Phys. Rev. D24 (1981) 3212.

[4] L. Caneschi, I. G. Halliday and A. Schwimmer, Nucl. Phys. B200 [FS4] (1982) 409.

[5] J. M. Alberty, H. Flyvbjerg and B. Lautrup, Nucl. Phys. B220[FS8] (1983) 61.

[6] M. C. Ogilvie, A. Horowitz, Nucl. Phys. B215[FS7] (1983) 249.

[7] R. Dashen, Urs M. Heller and H. Neuberger, Nucl. Phys. B215[FS7] (1983) 360.

[8] E. T. Tomboulis Phys. Lett. 303B (1993) 103.

[9] E. T. Tomboulis Phys. Lett. 108B (1982) 209.

[10] R. C. Brower, D. A. Kessler and H. Levine, Nucl. Phys. B205[FS5] (1982) 77.

[11] J. Fingberg, U. Heller and F. Karsch, Nucl. Phys. B392 (1993) 493.

[12] J. Engels, J. Fingberg and M. Weber, Nucl. Phys. B332 (1990) 737;

J. Engels, J. Fingberg and D. E. Miller, Nucl. Phys. B387 (1992) 501.

[13] B. Svetitsky and L. G. Yaffe, Nucl. Phys. B210[FS6] (1982) 423.

[14] R. V. Gavai, M. Grady and M. Mathur Nucl. Phys. B423 (1994) 123.

[15] M. Mathur, R. V. Gavai, Nucl. Phys. B448 (1995) 399; Nucl. Phys. B (PS) 42 (1995) 490.

[16] P. Stephenson, hep-lat/9509070.

[17] J. Engels and T. Scheideler, hep-lat/9610019.

[18] G. Bhanot and R. Dashen, Phys. Lett. 113B (1982) 299. 
[19] K.-H. Mütter and K. Schilling, Phys. Lett. 121B (1983) 267.

[20] R.V. Gavai, Nucl. Phys. B215 [FS7] (1983) 458.

[21] R.V. Gavai, F. Karsch and H. Satz, Nucl. Phys. B220 [FS8] (1983) 223.

[22] R.V. Gavai, Nucl. Phys. B474 (1996) 446

[23] A. Gonzalez-Arroyo and C. P. Korthals-Altes, Nucl. Phys. B205 (1982) 46.

[24] F. J. Wegner , J. Math. Phys. 12 (1971) 2259.

[25] L. McLerran and B. Svetitsky, Phys. Rev. D24 (1981) 450.

[26] M. N. Barber, in Phase Transitions and Critical Phenomena, vol. 8, Ed. C. Domb and J. L. Lebowitz (Academic Press, New York, 1983) p. 146.

[27] M. S. Challa, D. P. Landau and K. Binder, Phys. Rev. B34 (1986) 1841.

[28] J. Polonyi, K. Szlachanyi, Phys. Lett. 110B (1982) 395.

[29] F. Green, F. Karsch Nucl. Phys. B238 (1984) 297.

[30] F. Green, Nucl. Phys. B215 [FS 7] (1983) 83. 


\section{TABLES}

TABLE I. The average values of the critical exponent $\omega$ at different values of the adjoint couplings. The expected value is 1.97 (3.0) if the deconfining phase transition is second order (first order). The data taken from Refs. [12,14, 15]

\begin{tabular}{llr}
\hline \hline$\beta_{A}$ & $N_{\tau}$ & $\omega$ \\
\hline 0.0 & 4 & $1.93(03)$ \\
\hline 0.5 & 4 & $1.92(29)$ \\
\hline 0.75 & 4 & $1.53(32$ \\
\hline 0.9 & 4 & $2.10(22)$ \\
\hline 1.1 & 4 & $2.34(15)$ \\
\hline 1.4 & 2 & $3.246(243)$ \\
\hline \hline
\end{tabular}


TABLE II. The values of $\left(\beta, \beta_{A}\right)$ on $N_{\sigma}^{3} \times 2$ lattice at which simulations were performed, $\beta^{\text {crit. }}$ and the finite size scaling exponent $\omega$. The expected value for $\omega$ is 1.97 (3.0) if the deconfining phase transition is second order (first order).

\begin{tabular}{|c|c|c|c|c|}
\hline$\beta_{A}$ & $N_{\sigma}$ & $\beta$ & $\beta_{c}$ & $\omega$ \\
\hline \multirow{3}{*}{0.0} & 8 & 1.90 & 1.88 & \multirow{3}{*}{$1.92(01)$} \\
\hline & 10 & 1.885 & 1.878 & \\
\hline & 12 & 1.877 & 1.877 & \\
\hline \multirow{3}{*}{0.8} & 8 & 1.368 & 1.368 & \multirow{3}{*}{$2.03(01)$} \\
\hline & 10 & 1.367 & 1.3664 & \\
\hline & 12 & 1.368 & 1.366 & \\
\hline \multirow{3}{*}{0.9} & 8 & 1.3 & 1.31 & \multirow{3}{*}{$1.83(02$} \\
\hline & 10 & 1.3 & 1.31 & \\
\hline & 12 & 1.3092 & 1.3088 & \\
\hline \multirow{3}{*}{1.1} & 8 & 1.201 & 1.201 & \multirow{3}{*}{$1.79(02$} \\
\hline & 10 & 1.2 & 1.2 & \\
\hline & 12 & 1.1999 & 1.1995 & \\
\hline \multirow{4}{*}{1.25} & 8 & 1.12 & 1.1203 & \multirow{4}{*}{$1.97(01)$} \\
\hline & 10 & 1.12 & 1.1203 & \\
\hline & 12 & 1.12 & 1.12 & \\
\hline & 16 & 1.12 & 1.1196 & \\
\hline
\end{tabular}


TABLE III. The values of $\left(\beta, \beta_{A}\right)$ on $N_{\sigma}^{3} \times 4$ lattice at which simulations were performed, $\beta^{\text {crit. }}$ and the finite size scaling exponent $\omega$.

\begin{tabular}{lcccc}
\hline \hline$\beta_{A}$ & $N_{\sigma}$ & $\beta$ & $\beta_{c}$ & $\omega$ \\
\hline & 8 & 1.327 & 1.327 & \\
1.1 & 10 & 1.327 & 1.3274 & \\
& 12 & 1.32715 & 1.32715 & \\
\hline & 8 & 1.2146 & 1.2142 & $3.13(01)$ \\
1.25 & 10 & 1.214 & 1.2144 & \\
\hline \hline
\end{tabular}




\section{FIGURE CAPTIONS}

Fig. 1 The phase diagram of the extended SU(2) lattice gauge theory. The solid lines are from simulations done on a $5^{4}$ lattice by Bhanot and Creutz [i]]. The light dashed line indicates the absence of first order bulk transition [22] below $\beta_{A}=1.25$. The dotted(thick dashed) lines with hollow(filled) symbols are the second(first) order deconfinement phase transition lines on $N_{\tau}=2$ (circles) and 4 (squares) lattices.

Fig. 2 Polyakov loop susceptibility at (a) $\beta_{A}=0.0$, (b) $\beta_{A}=0.8$, (c) $\beta_{A}=1.1$ and (d) $\beta_{A}=1.25$ on $8^{3} \times 2,10^{3} \times 2$ and $12^{3} \times 2$ lattices. At $\beta_{A}=1.25$ result on $16^{3} \times 2$ lattice is also shown. The points with error bars are results of simulations and the curves are extrapolations by the histogramming technique. The horizontal lines are predictions assuming a second order deconfinement transition, as explained in the text.

Fig. 3 Evolution of $|L|$ and $P$ at $\beta_{A}=1.25$ on (a) $12^{3} \times 4(\beta=1.2144)$, (b) $10^{3} \times 4(\beta=1.214)$ and $(\mathrm{c}) 8^{3} \times 4(\beta=1.2146)$ lattices.

Fig. 4 Polyakov loop susceptibility at (a) $\beta_{A}=1.1$ and (b) $\beta_{A}=1.25$ on $8^{3} \times 4,10^{3} \times 4$ and $12^{3} \times 4$ lattices. The solid horizontal lines are predictions assuming a first (second) order deconfinement transition at $\beta_{A}=1.25\left(\beta_{A}=1.1\right)$, as explained in the text. The broken lines in Fig. 4a are predictions for second order.

Fig. 5

The probability density of $\mathrm{L}$ at $\beta_{A}=1.25$ on $12^{3} \times 6$ lattice at $\beta=1.2184$. Fig. 6

The probability density of L at $\beta_{A}=1.1$ on $N_{\sigma}^{3} \times N_{\tau}$ lattice for a] $N_{\tau}=4, N_{\sigma}=8,10,12$ and $\beta=1.327,1.327$ and 1.32685 respectively, b] $N_{\tau}=6, N_{\sigma}=12$ and $\left.\beta=1.339, \mathrm{c}\right] N_{\tau}=8$, $N_{\sigma}=16$ and $\beta=1.35$ and 1.3508 . 


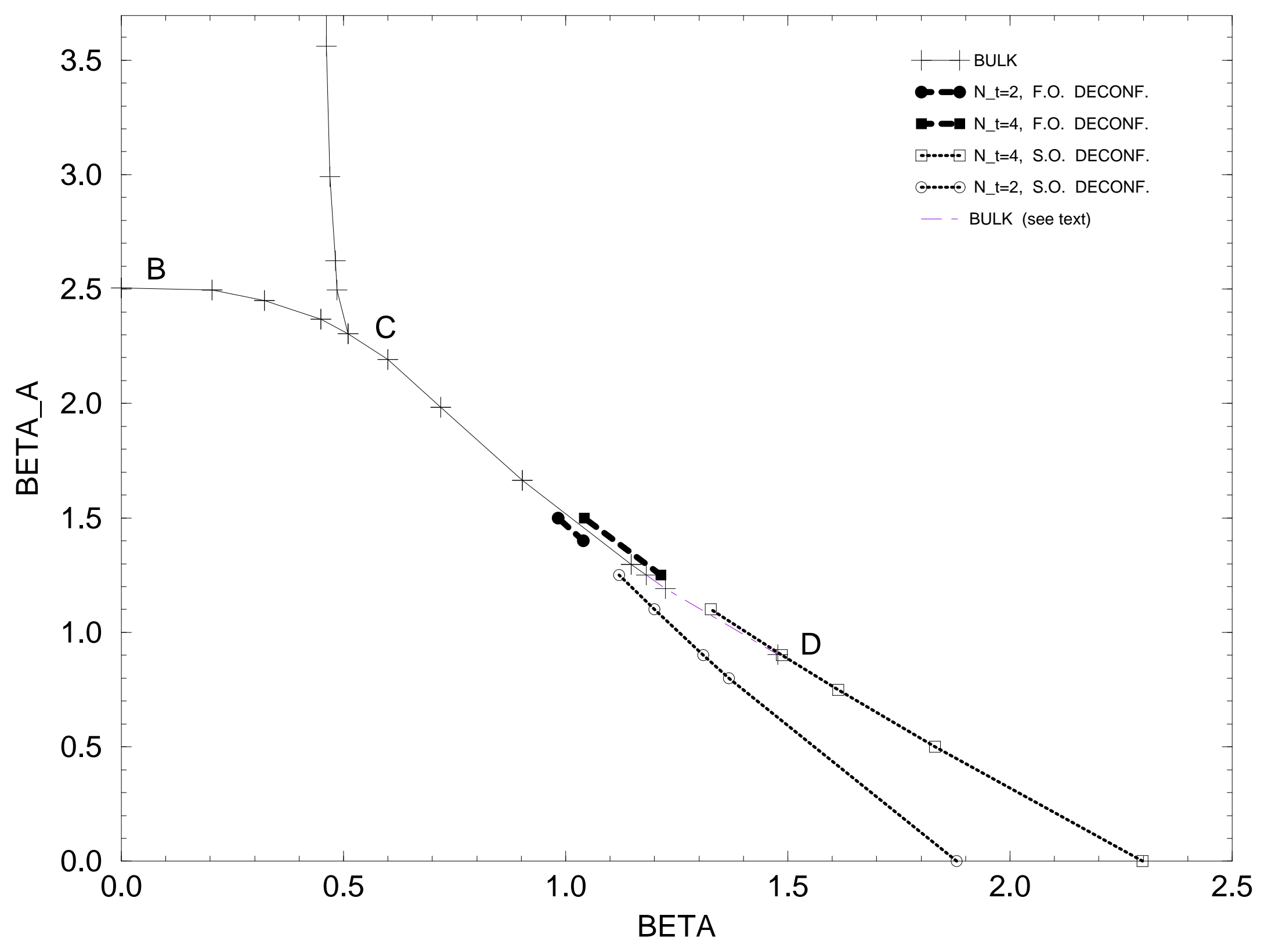



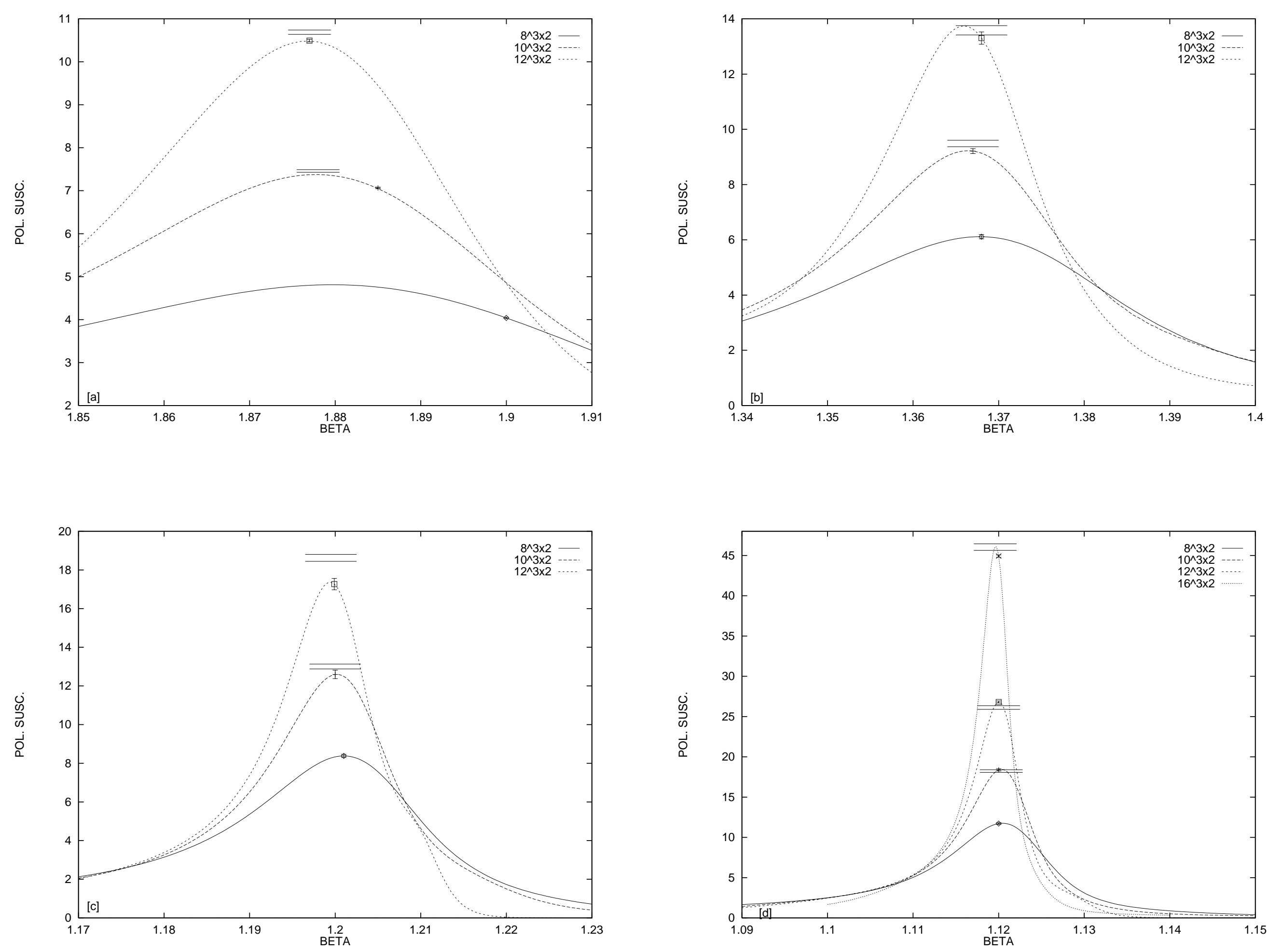

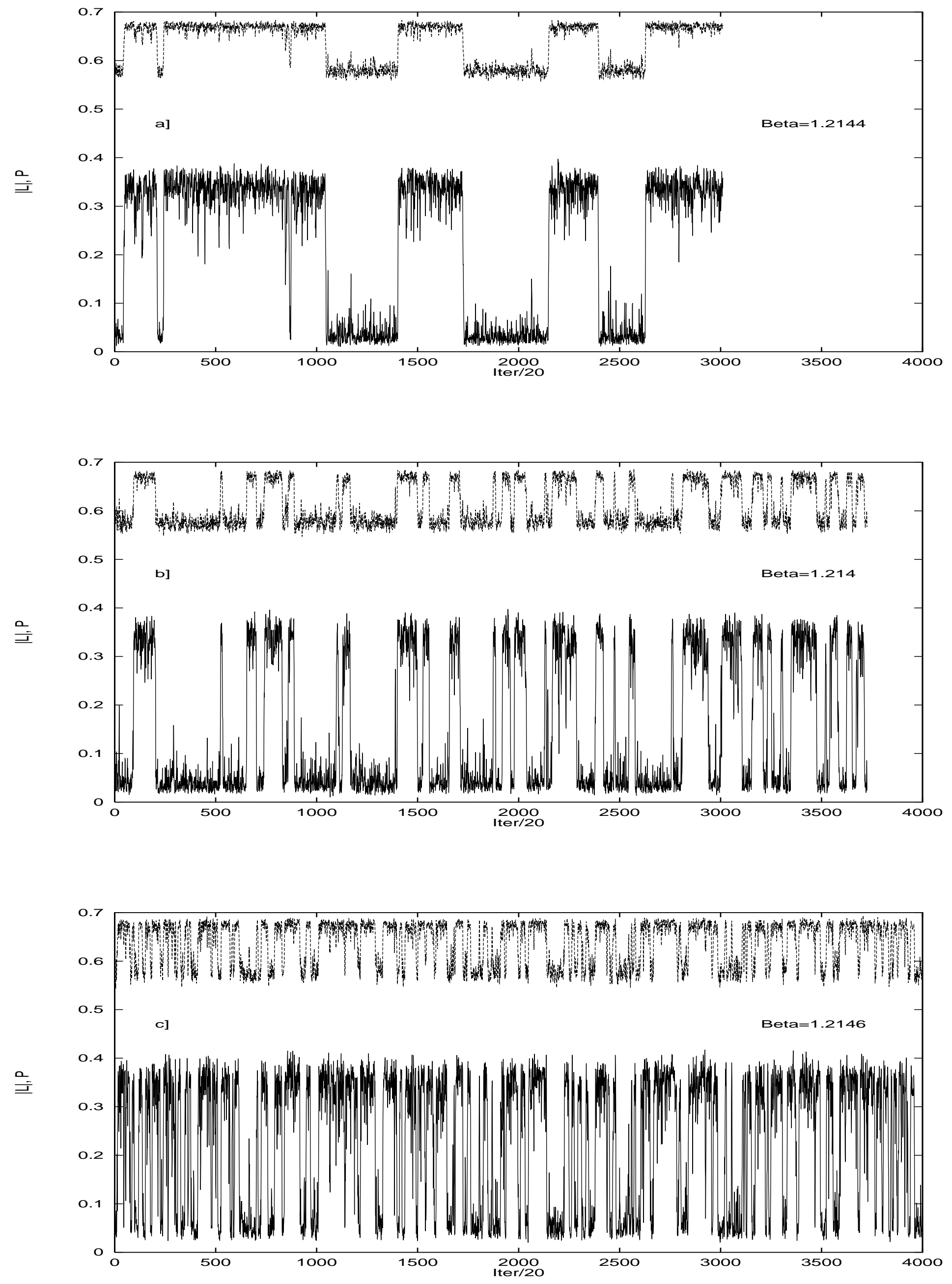

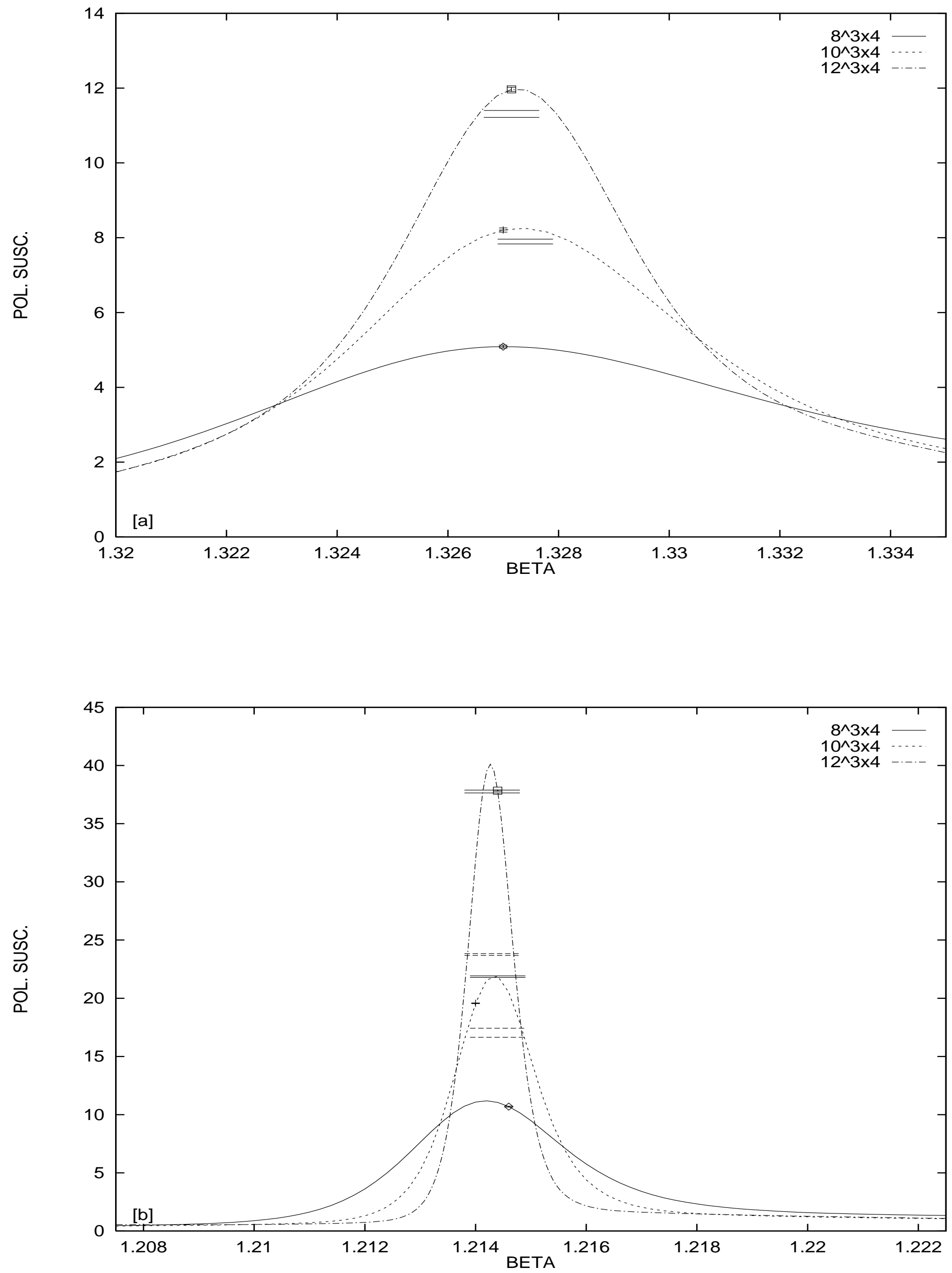


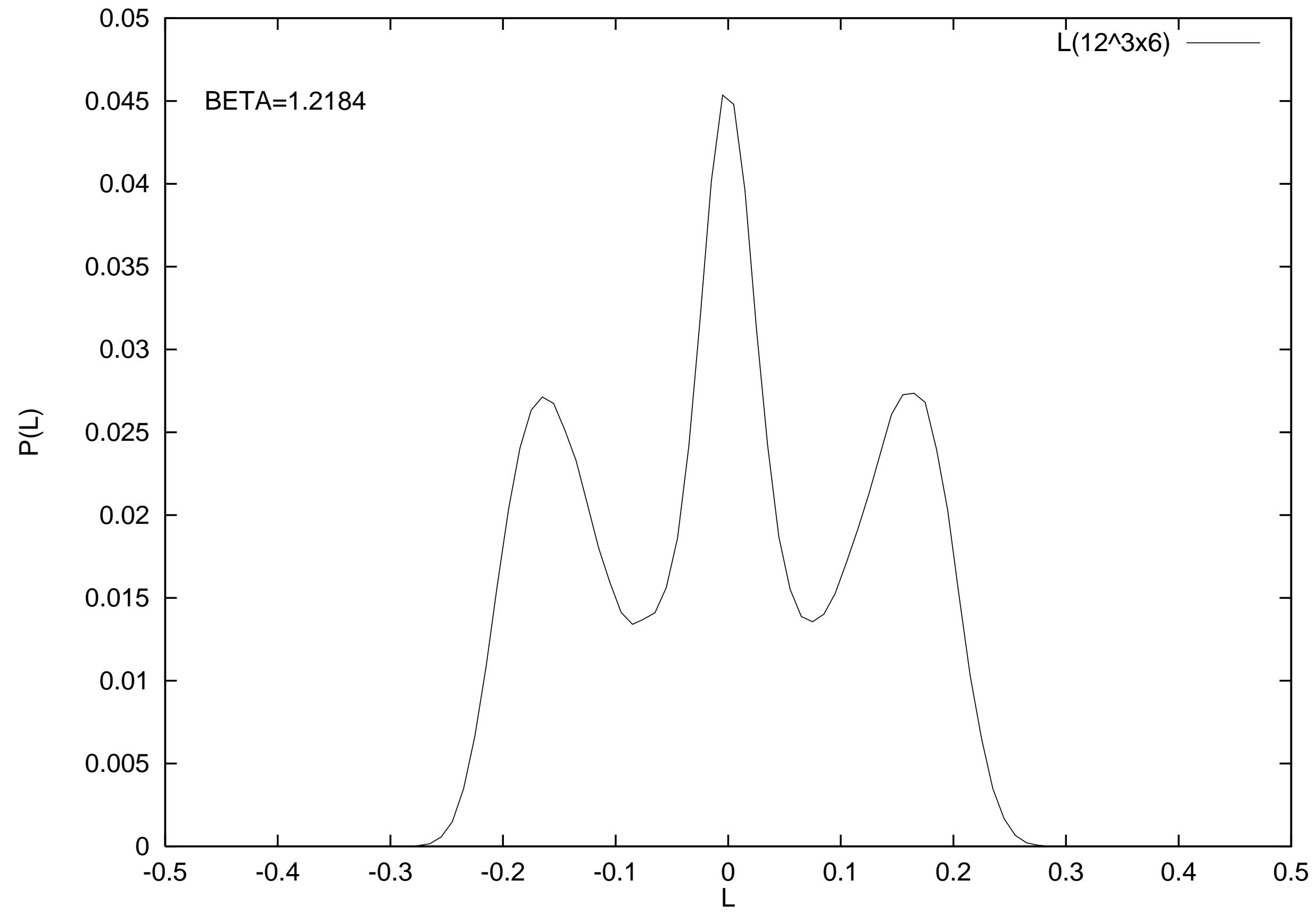




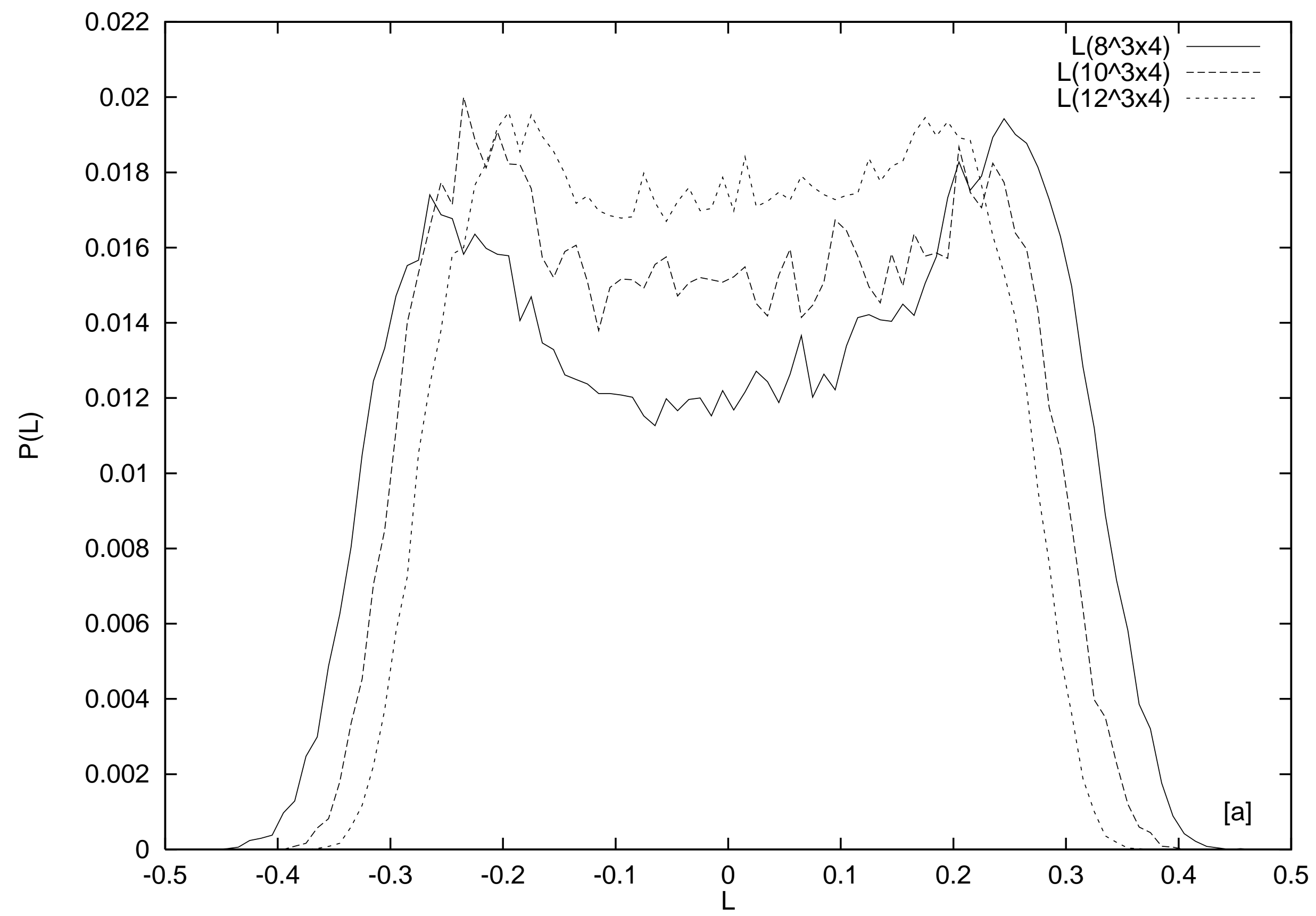




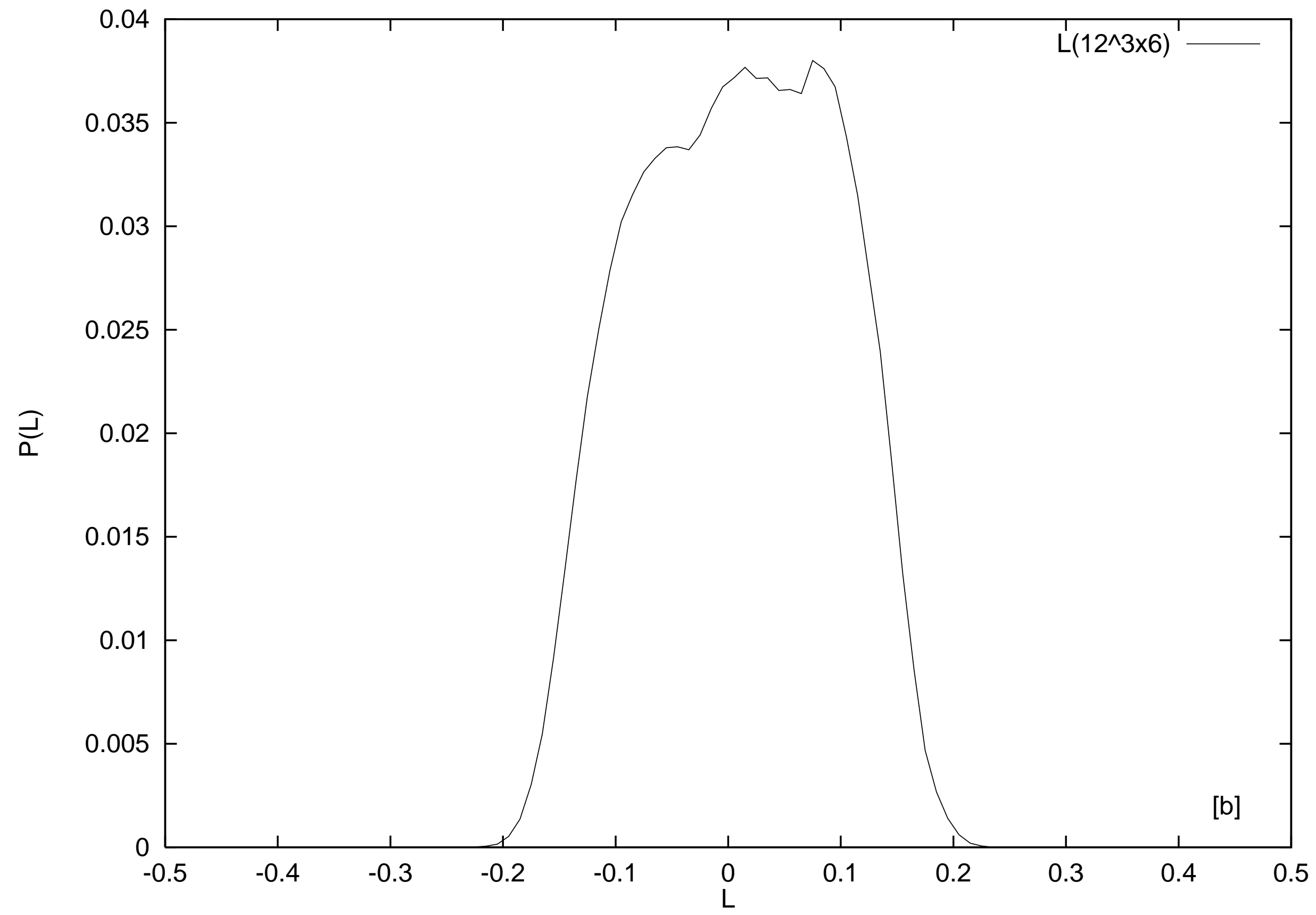




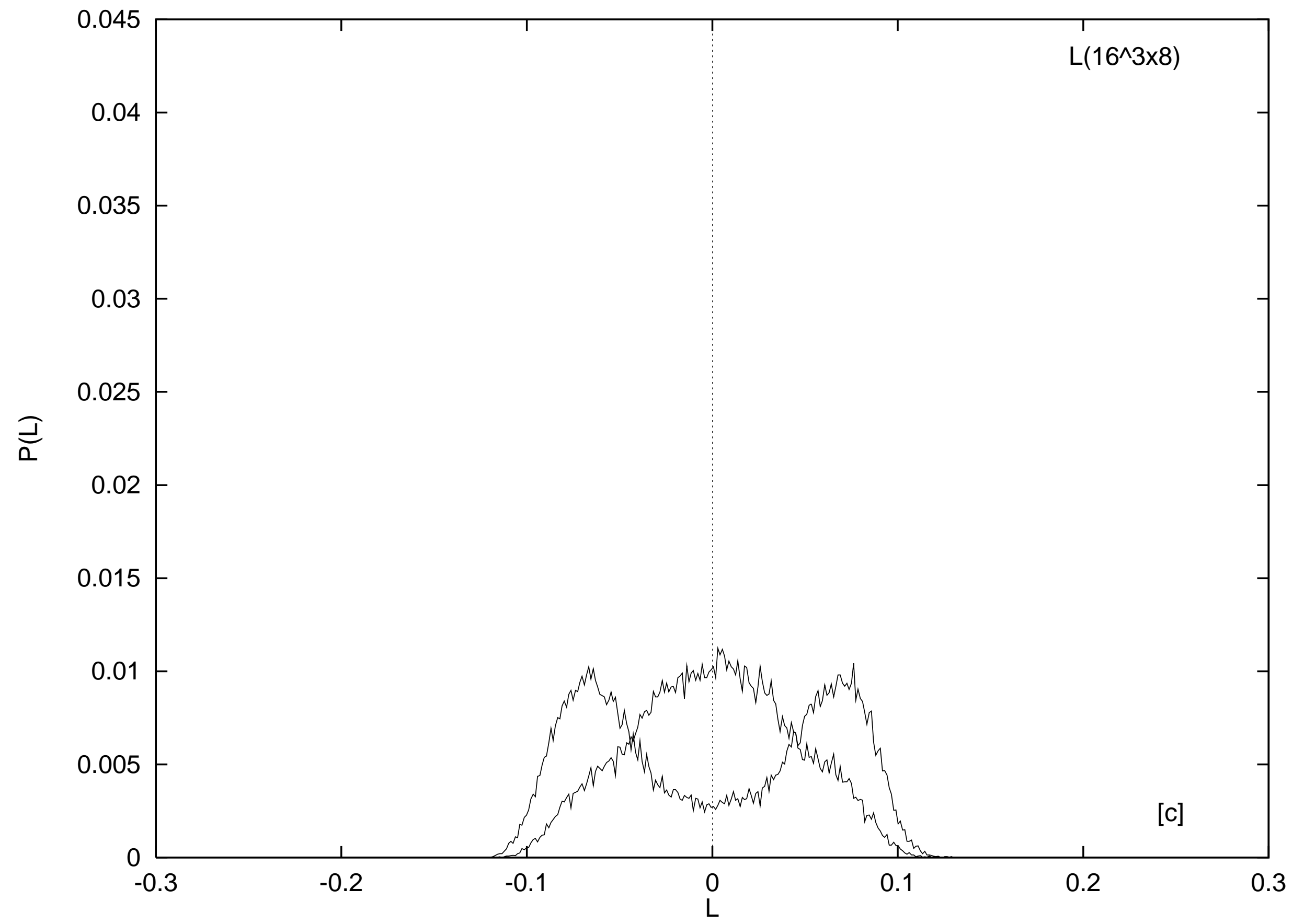

